\title{
The unusual magnetism of nanoparticle $\mathrm{LaCoO}_{3}$
}

\author{
A. M. Durand, ${ }^{1}$ D. P. Belanger, ${ }^{1}$ T. J. Hamil,${ }^{1}$ F. Ye, ${ }^{2}$ S. Chi, ${ }^{2}$ \\ J. A. Fernandez-Baca, ${ }^{2}$ C. H. Booth, ${ }^{3}$ Y. Abdollahian, ${ }^{4}$ and M. Bhat ${ }^{5}$ \\ ${ }^{1}$ Department of Physics, University of California, Santa Cruz, CA 95064, USA \\ ${ }^{2}$ Quantum Condensed Matter Division, Oak Ridge National Laboratory, Oak Ridge, Tennessee 37831, USA \\ ${ }^{3}$ Chemical Sciences Division, Lawrence Berkeley National Laboratory, Berkeley, CA 94720, USA \\ ${ }^{4}$ Department of Chemistry, University of California, Santa Cruz, CA 95064, USA \\ ${ }^{5}$ Castilleja School, Palo Alto, CA 94301, USA
}

(Dated: May 16, 2022)

\begin{abstract}
Bulk and nanoparticle powders of $\mathrm{LaCoO}_{3}$ (LCO) were synthesized, and their magnetic and structural properties were studied using SQUID magnetometry and neutron diffraction. The bulk and large nanoparticles exhibit weak ferromagnetism $(\mathrm{FM})$ below $T \approx 85 \mathrm{~K}$ and a crossover from strong to weak antiferromagnetic (AFM) correlations near a transition expressed in the lattice parameters, $T_{o} \approx 40 \mathrm{~K}$. This crossover does not occur in the smallest nanoparticles; instead, the magnetic behavior is predominantly ferromagnetic. The amount of FM in the nanoparticles depends on the amount of $\mathrm{Co}_{3} \mathrm{O}_{4}$ impurity phase, which induces tensile strain on the LCO lattice. A coreinterface model is introduced, with the core region exhibiting the AFM crossover and with FM in the interface region near surfaces and impurity phases.
\end{abstract}

PACS numbers:

The unusual magnetic behavior of $\mathrm{LaCoO}_{3}(\mathrm{LCO})$ has remained largely unexplained, despite the growing realization that structural distortion represents an important degree of freedom influencing the behavior of a large class of perovskites [1, 2]. Recently, strain-switched ferromagnetism (FM) in LCO has been used to create a spintronic device. 3] Although the temperature at which that device operates is low $(T<90 \mathrm{~K})$, understanding the mechanism behind strain-induced LCO magnetism should facilitate the search for similar perovskite materials that will allow switching of the ferromagnetic moment at higher temperatures. Finding such a material will allow construction of spintronic devices for widespread use. Recently, a model for the magnetism was developed [4] that explains the crucial role that the $\mathrm{Co}_{3} \mathrm{O}_{4}$ impurity phase plays in the formation of long-range ferromagnetic order in LCO. The model involves two regions: the interface region near the boundaries between the $\mathrm{LCO}$ and $\mathrm{Co}_{3} \mathrm{O}_{4}$ phases as well as near the LCO particle surfaces, and the core LCO region away from these interfaces and surfaces. In this work, we apply the model to explain the effects of the particle surfaces and $\mathrm{Co}_{3} \mathrm{O}_{4}$ impurity phase on the LCO magnetism; these effects are more pronounced as the LCo particle size decreases to the nanoscale.

Many earlier attempts to model LCO magnetism focused on local transitions between Co electron states. Such models do not provide a comprehensive description of the variety of phenomena observed in films, bulk and nanoparticle powders, and single crystals of LCO. More recent efforts recognize the importance of including collective behaviors of the correlated electrons. [5- -7$]$ By considering four samples with different sized particles and $\mathrm{Co}_{3} \mathrm{O}_{4}$ impurity phase concentrations, we show that the disparate magnetic behaviors observed in various LCO systems fit well into the model developed [4] for the bulk particles.

\section{Synthesis and Characterization}

The LCO bulk sample, A, was synthesized using a standard solid state reaction [8]. Stoichiometric amounts of $\mathrm{La}_{2} \mathrm{O}_{3}$ and $\mathrm{Co}_{3} \mathrm{O}_{4}$ were ground together thoroughly and fired for 8 hours. This process was repeated five times, with firing temperatures between $850^{\circ} \mathrm{C}$ to $1050^{\circ} \mathrm{C}$.

LCO nanoparticles were synthesized using the amorphous heteronuclear complex DTPA. [9, 10] A $1.0 \mathrm{M}$ $\mathrm{NaOH}$ solution was added by drops to an aqueous solution of $\mathrm{La}\left(\mathrm{NO}_{3}\right)_{3} \cdot 6 \mathrm{H}_{2} \mathrm{O}$ and $\mathrm{Co}\left(\mathrm{NO}_{3}\right)_{3} \cdot 6 \mathrm{H}_{2} \mathrm{O}$ to prepare hydroxides. A stoichiometric amount of $\mathrm{NaOH}$ was used for sample $\mathrm{D}$, and excess $\mathrm{Na}$ ions were removed via dialysis over 24 hours. This resulted in significant $\mathrm{Co}_{3} \mathrm{O}_{4}$ phase, likely as some La ions were removed along with the Na. Only a $12.5 \%$ stoichiometric amount of $\mathrm{NaOH}$ was added to samples $\mathrm{B}$ and $\mathrm{C}$, and no dialysis was undertaken, resulting in less $\mathrm{Co}_{3} \mathrm{O}_{4}$. For all cases, equimolar amounts of DTPA were then added to the metal hydroxides, and the resulting complex precursor was stirred while heated to $80^{\circ} \mathrm{C}$. The resulting transparent solution was vaporized slowly at $80^{\circ} \mathrm{C}$ until a dark purple resinlike gel formed, which was decomposed in air at $350^{\circ} \mathrm{C}$ for 1.5 hours. The ash-like material was then calcined for 4 hours at $620^{\circ} \mathrm{C}$ for samples $\mathrm{C}$ and $\mathrm{D}$ and $1000^{\circ} \mathrm{C}$ for sample B.

X-ray scattering data for characterization were taken using a Rigaku SmartLab powder diffractometer equipped with a copper x-ray tube $\left(\lambda\left(\mathrm{Cu} \mathrm{K}_{\alpha}\right)=1.54056\right.$ $\AA$, tube energy $44 \mathrm{~mA} / 40 \mathrm{kV}$ ). The samples were analyzed with a scan rate of $3.0^{\circ} / \mathrm{min}$ with a step size of $0.02^{\circ}$. Scans showed that all samples were predom- 
TABLE I: Synthesis characterizations for samples A, B, C, and D. The methods are described in the text for the bulk (1), nanoparticle (2), and nanoparticle with dialysis (3). $T_{\text {form }}$ is the temperature at which the particles formed. The determinations of the particle sizes and $\mathrm{Co}_{3} \mathrm{O}_{4}$ weight percentages are described in the text.

\begin{tabular}{|c|c|c|c|c|}
\hline & $\overline{\text { Method }}$ & $T_{\text {form }}\left({ }^{\circ} \mathrm{C}\right)$ & Size (nm) & $\% \mathrm{Co}_{3} \mathrm{O}_{4}$ \\
\hline $\bar{A}$ & 1 & $850-1050$ & $\approx 500$ & 4.5 \\
\hline B & 2 & 1000 & $100-400$ & 0 \\
\hline $\mathrm{C}$ & 2 & 620 & 18 & 11 \\
\hline $\mathrm{D}$ & 3 & 620 & 22 & 28 \\
\hline
\end{tabular}

inantly LCO phase, and contained varying amounts of the $\mathrm{Co}_{3} \mathrm{O}_{4}$ phase; the latter were further quantified in the neutron scattering measurements. Samples $\mathrm{C}$ and $\mathrm{D}$ were also examined using small angle $\mathrm{x}$-ray scattering (SAXS) on the same x-ray diffractometer. The nanoparticles were placed in between two layers of scotch tape, and transmission SAXS was performed. Particle size and distribution data were analyzed using the NANO-solver software included with the diffractometer.

The particles in A and B were too large for size determinations using either neutron or x-ray scattering. We estimate an average size of $500 \mathrm{~nm}$ for $\mathrm{A}$ and between 100 and $400 \mathrm{~nm}$ for B based on TEM measurements on a bulk powder and a similar $\mathrm{La}_{1-\mathrm{x}} \mathrm{Sr}_{\mathrm{x}} \mathrm{CoO}_{3}$ nanoparticle powder with a calcination temperature of $1000^{\circ} \mathrm{C}$, respectively.

Standard Scherrer analysis of x-ray diffraction data for sample D yielded average crystallite sizes of $22 \mathrm{~nm}$. SAXS measurements on the same sample yielded an average particle size of $65 \mathrm{~nm}$, suggesting the agglomeration of LCO nanoparticle crystallites during growth. Scherrer analysis for sample $\mathrm{C}$ gave an average crystallite size of $18 \mathrm{~nm}$, and the SAXS gave an average agglomerate size of $53 \mathrm{~nm}$.

\section{Neutron Diffraction}

Figure 11 shows the neutron powder diffraction intensity vs $2 \theta$ at $T=10 \mathrm{~K}$ from the WAND instrument at ORNL, along with FullProf refinements [11] done using the $\mathrm{R} \overline{3} \mathrm{c}$ symmetry for LCO. The distribution of small, non-spherical particles makes nanoparticle refinements more challenging. Bulk and nanoparticle powders exhibit small $\mathrm{Co}_{3} \mathrm{O}_{4}$ and $\mathrm{CoO}$ phase peaks with $\mathrm{F} \overline{4} 3 \mathrm{~m}$ and Fm3m symmetries, respectively. The $\mathrm{Co}_{3} \mathrm{O}_{4}$ structural and magnetic peaks were of high enough intensity to be refined by FullProf; the calculated weight percentages are shown in Table I. The relatively sharp Bragg peaks seen for this phase indicate that the $\mathrm{Co}_{3} \mathrm{O}_{4}$ forms crystallites, which are likely interspersed within the LCO nanoparticles and bulk. As only the $\mathrm{CoO}$ high intensity magnetic peak could be seen, refinements were not possible on this phase. It is therefore unlikely that there is a significant

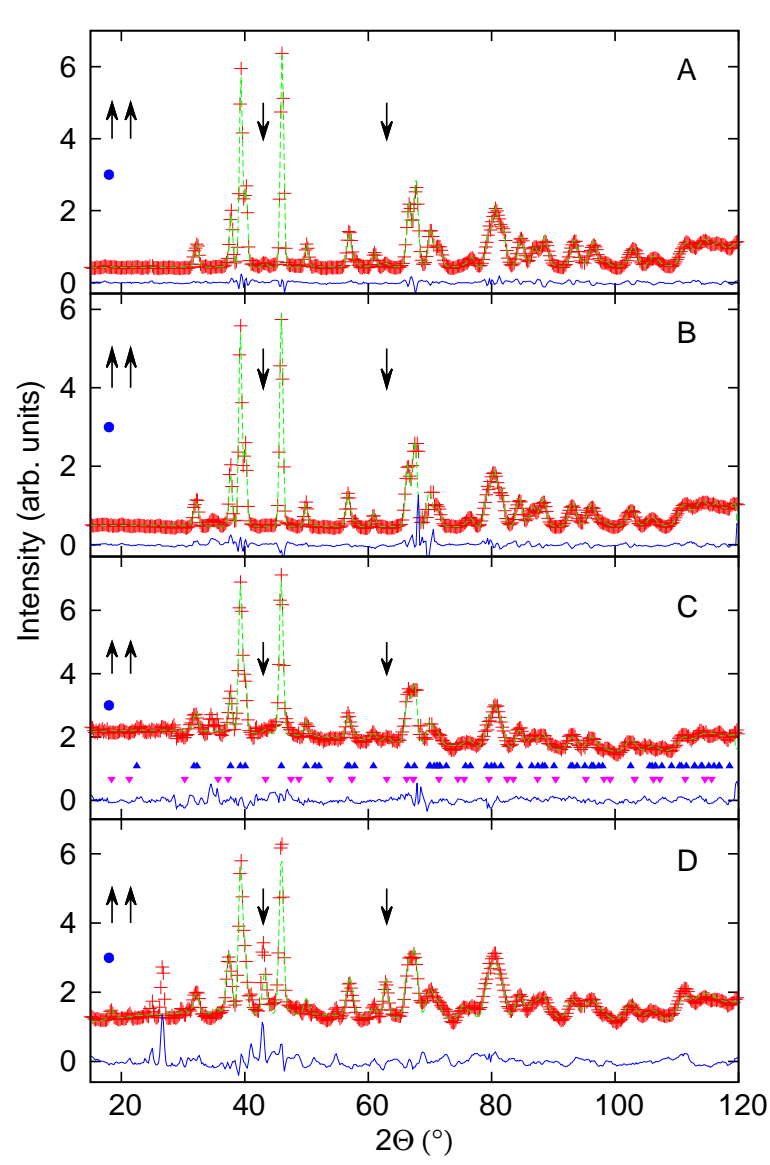

FIG. 1: Neutron diffraction intensity vs $2 \theta$ for $T=10 \mathrm{~K}$ with FullProf refinements using the $\mathrm{R} \overline{3} \mathrm{c}$ perovskite structure as well as the difference between the fit and calculation (lower curve) for samples A, B, C, and D. The upper row of triangles in panel $\mathrm{C}$ indicates calculated peak positions for $\mathrm{LCO}$ and the lower row of inverted triangles indicates peak positions for $\mathrm{Co}_{3} \mathrm{O}_{4}$. The down arrows indicate observed $\mathrm{Co}_{3} \mathrm{O}_{4}$ structural peaks and the up arrows indicate $\mathrm{Co}_{3} \mathrm{O}_{4}$ magnetic peaks. The filled dot indicates the magnetic peak position for $\mathrm{CoO}$.

amount of $\mathrm{CoO}$ in these samples; in addition to the neutron scattering data, no indication is given in the magnetometry data of a significant $\mathrm{CoO}$ magnetic moment as was seen in the samples of Fita et al. [12]

The bulk (A) average lattice parameter $a(T)$, shown in Fig. 2. is significantly smaller than that of the nanoparticles (B, C and D). For $T>T_{o}, \mathrm{~A}$ and $\mathrm{B}$ show significant curvature, whereas $\mathrm{C}$ shows only slight curvature and $\mathrm{D}$ shows no significant curvature. The inset of Fig. 2 shows the $T=30 \mathrm{~K}$ values of $\gamma(T)$ (the Co-O-Co bond angle) and $\delta y(T)=\frac{d}{a} \cos (\gamma / 2)$, where $d$ is the Co-O bond length. The parameter $\delta y(T)$ characterizes the rhombohedral distortion of the lattice [5 7, 13]. The parameters $\gamma$ and $\delta y$ are nearly proportional because $d$ varies by less than $0.4 \%$ across the samples at low $T$.

The lattice parameter $a(T)$ for samples A, B and C, 


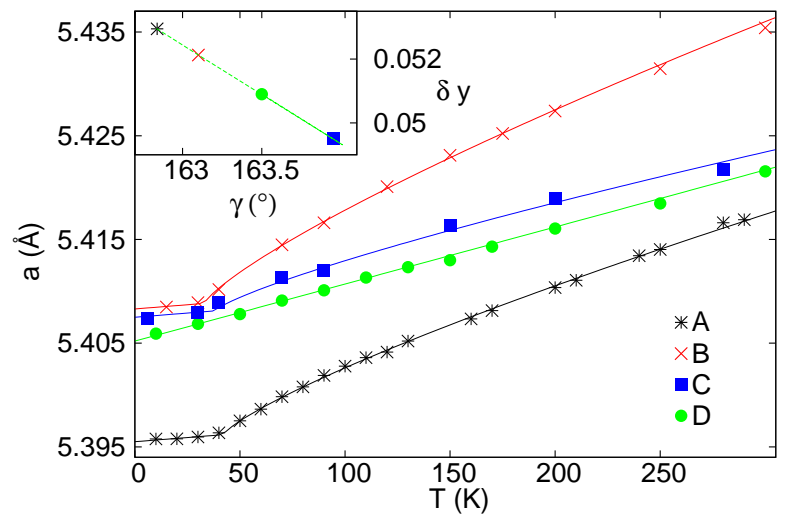

FIG. 2: Lattice parameter $a(T)$ vs T for A, B, C and D. The inset shows $\delta y$ vs $\gamma$ for the same samples at $T=30 \mathrm{~K}$.

TABLE II: Fit parameters using Eq. 1 and 2 with fixed exponent $\sigma=0.8$. Errors in $\alpha$ and $K_{a}$ are similar for all samples.

\begin{tabular}{lcccc}
\hline & $\mathrm{A}$ & $\mathrm{B}$ & $\mathrm{C}$ & $\mathrm{D}$ \\
\hline$a_{0}(\AA)$ & $5.396(1)$ & $5.409(1)$ & $5.406(1)$ & $5.407(1)$ \\
$T_{o}(K)$ & $42(2)$ & $33(2)$ & $38(2)$ & - \\
$\alpha$ & $3.0(5) \times 10^{-6}$ & $3.0 \times 10^{-6}$ & $3.0 \times 10^{-6}$ & $5.5 \times 10^{-5}$ \\
$K_{a}$ & $4.97(2) \times 10^{-3}$ & $5.10 \times 10^{-3}$ & $3.27 \times 10^{-3}$ & - \\
\hline
\end{tabular}

was fitted using

$$
a(T)=a_{0}(1+\alpha T) \quad\left(T<T_{o}\right)
$$

and

$$
a(T)=a\left(T_{o}\right)+K_{a}\left(\frac{T-T_{o}}{T_{o}}\right)^{\sigma} \quad\left(T>T_{o}\right),
$$

with the parameters in Table II. The temperature $T_{o}$ is the crossover point for the linear and power law behaviors, and has previously been suggested as the critical temperature for a collective phase transition in bulk LCO. [4] Although more data are needed in the vicinity of the transition to ascertain the true value of $T_{o}$ in the $\mathrm{B}$ and $\mathrm{C}$ nanoparticle samples, we note that the data are in qualitative agreement with the bulk LCO results. The data shown in Fig. 2 were fit with values of $T_{o}$ ranging from 33 to $42 \mathrm{~K}$. The sharp change in slope at $T_{o}$ is also observed in the lattice parameter $c(T)$ for $\mathrm{A}, \mathrm{B}$, and $\mathrm{C}$. Sample D is better fit to Eq. 1 1 over the entire $T$ range.

\section{Magnetometry}

The behavior of the magnetization in LCO has long been considered unusual and yet no adequate model of it below room temperature has been developed. Local Co spin-state models predict a nonmagnetic ground state because there are no moments when the spins are paired in the lowest energy state. Moments are then thought to develop as spins are thermally excited near $T=90 \mathrm{~K}$. However, our data indicate that the ground state for material away from the interfaces and particle surfaces clearly has magnetic moments at low temperature, though they do not order. Regions close to the interfaces or surfaces develop ferromagnetic order at low temperature. Recent work has shown the importance of extended states [6] in LCO bulk particles, a FM phase transition in LCO at $T_{c} \approx 87 \mathrm{~K}$, and another transition near $T_{o} \approx 40 \mathrm{~K}$. [5] These behaviors, as well as others reported for bulk and nanoparticle powders and thin films, [14 17] are consistent with a particle core-interface model that includes, for particles larger than $\approx 20 \mathrm{~nm}$, a core region exhibiting a crossover between two types of paramagnetism near $T_{o}$, and an interface region located near surfaces or interfaces with impurity phases. Tensile stress, either from the lattice mismatch between the interfaces and the core, or between the particle surface and the core, can induce a FM transition in the interface region below $T_{c}$. 16, 18]

Figures 3 and 4 show $M / H$ and $H / M$ vs $T$, respectively, for the $\mathrm{LaCoO}_{3}$ bulk and nanoparticle powders upon field cooling. Comprehensive fits using a simple superposition of FM and AFM behaviors were not successful. Instead, samples A, B and D were successfully fit using a superposition of two different Curie-Weiss-like $(\mathrm{CW})$ paramagnetic behaviors and one power-law FM behavior,

$$
\begin{gathered}
\frac{M}{H}(T)=\left(d+\frac{E_{a}}{T+t_{a}}\right) S(T)+\left(\frac{E_{b}}{T+t_{b}}\right) \\
\quad+M_{n}\left(\frac{T_{c}-T}{T_{c}}\right)^{\beta}(S(T)+L(1-S(T))),
\end{gathered}
$$

for $T \leq T_{C}$ and

$$
\frac{M}{H}(T)=\left(d+\frac{E_{a}}{T+t_{a}}\right) S(T)+\left(\frac{E_{b}}{T+t_{b}}\right)
$$

for $T \geq T_{C}$, where the subscript $n$ indicates the field in Oe. Each term is modified by a sigmoid, centered at temperature $T_{S}$ with a width $1 / W$,

$$
S(T)=\frac{1}{1+\exp \left(W\left(T_{S}-T\right)\right)} .
$$

This expression for $\frac{M}{H}(T)$ captures a crossover in the paramagnetic behavior at high $T$ (with parameters $E_{a}$ and $\left.t_{a}\right)$ to a low $T$ behavior $\left(E_{b}\right.$ and $\left.t_{b}\right)$ as $T$ decreases. While the Curie-Weiss fits work well over the limited $T$ range used, the $E$ and $t$ parameters differ from high $T$ fits over the wide range $170<T<300 \mathrm{~K}$ for the bulk. [5] The parameters cannot be directly interpreted as yielding the moment and interaction strength because the usual interpretation of the Curie-Weiss expression presumes weakly 


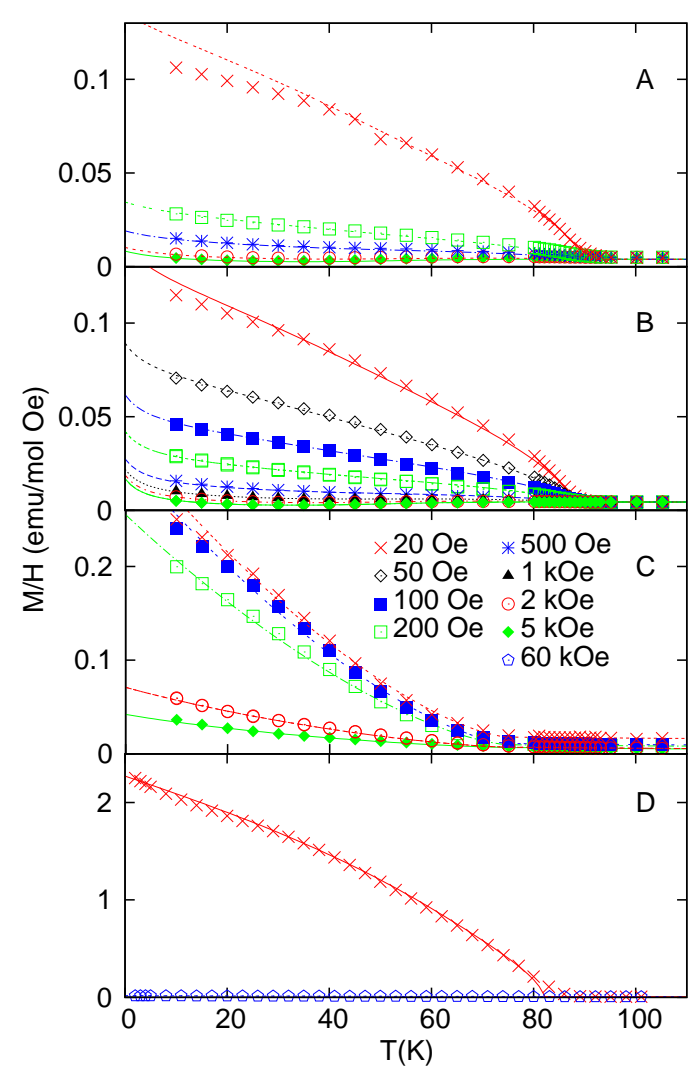

FIG. 3: $M / H$ vs. $T$ for samples A, B, C and D for fields $20<H<60000$ Oe along with fits described in the text. Note the different vertical scales for each data set.

interacting moments. For sample $\mathrm{C}$, which shows no evidence for a crossover or sharp transition to ferromagnetic order, but significant variation above $T_{c}$, data were fit to

$$
\frac{M}{H}(T)=d_{n}+\frac{E_{a}}{T+t_{a}}+M_{n}\left(\frac{T_{c}-T}{T_{c}}\right)^{\beta},
$$

where the exponent for the power law is $\beta=1.5$. For this sample, the parameter $d$ varied with the field. For the fit to the data for sample $\mathrm{D}, t_{a}$ and $t_{b}$ were set equal.

Despite the large number of parameters, shown in Table III] at a given $H$, the fits are strongly constrained because only $M$ was allowed to vary with $H$ for samples $\mathrm{A}, \mathrm{B}$ and $\mathrm{D}$, and only $M$ and $d$ were allowed to vary for sample $\mathrm{C}$. The $H$-dependent parameters characterize the FM contributions. AFM contributions, associated with the CW functions, are expected to be insensitive to $H$ and dominate for large $H$. They are most apparent for $H / M$ vs $T$ in Fig. 4 . The FM power law is most dominant at small $H$ and is best seen in Fig. 3. The FM fixed point is at $H=0$, so the power law behavior for $M / H$ vs $T$ is stronger and sharper as $H$ decreases.

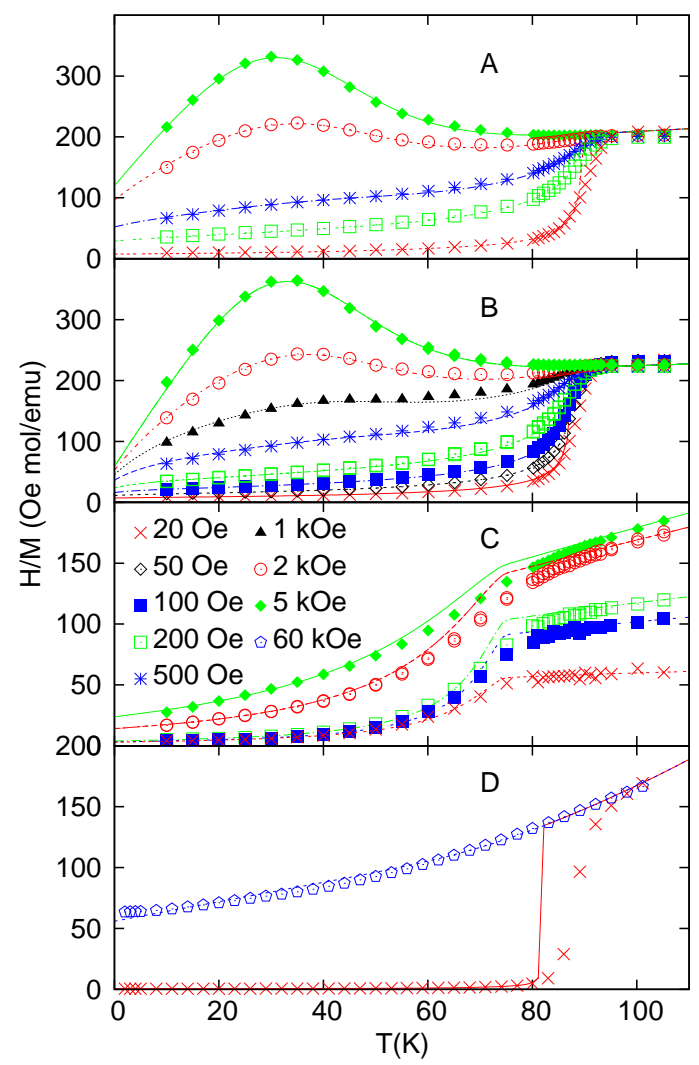

FIG. $4: H / M$ vs. $T$ for the same data and fits shown in Fig. 3

TABLE III: Fit parameters for samples A, B, C, and D using Eq. 3 and 6 with fixed value $\beta=0.63$. The parameter $d$ varies with $H$ only for sample $\mathrm{C}$, for which values are listed in the last row. The $M$ and $d$ subscripts refer to the applied field in Oe.

\begin{tabular}{lllll}
\hline & $\mathrm{A}$ & $\mathrm{B}$ & $\mathrm{C}$ & $\mathrm{D}$ \\
\hline$T_{C}(\mathrm{~K})$ & $89.5(5)$ & $87.5(5)$ & $75(2)$ & $82(5)$ \\
$T_{S}(\mathrm{~K})$ & $49(2)$ & $47(2)$ & $50(10)$ & $80(20)$ \\
$W(1 / \mathrm{K})$ & $0.09(1)$ & 0.09 & 0.09 & 0.05 \\
$t_{a}(\mathrm{~K})$ & $180(20)$ & $70(5)$ & $40(5)$ & $80(20)$ \\
$t_{b}(\mathrm{~K})$ & $11.5(5)$ & $5(1)$ & - & $80(20)$ \\
$E_{a}(\mathrm{erg} \mathrm{K} / \mathrm{mol} \mathrm{Oe})$ & $0.60(2)$ & $0.15(5)$ & $0.73(5)$ & $0.88(5)$ \\
$E_{b}(\mathrm{erg} \mathrm{K} / \mathrm{mol} \mathrm{Oe})$ & $0.094(1)$ & $0.077(1)$ & - & $1.5(1)$ \\
$L$ & $1.2(2)$ & $1.15(2)$ & 1 & 1.4 \\
$d_{(\mathrm{erg} / \mathrm{mol} \mathrm{Oe})}$ & $0.0019(2)$ & $0.0036(1)$ & - & $0.0004(1)$ \\
$M_{20}(\mathrm{erg} / \mathrm{mol} \mathrm{Oe})$ & $0.106(3)$ & $0.11(1)$ & $0.31(1)$ & $1.61(2)$ \\
$M_{50}$ & - & $0.063(2)$ & - & - \\
$M_{100}$ & - & $0.039(2)$ & $0.29(1)$ & - \\
$M_{200}$ & $0.022(2)$ & $0.022(2)$ & $0.23(1)$ & - \\
$M_{500}$ & $0.0092(3)$ & $0.0092(5)$ & - & - \\
$M_{1000}$ & - & $0.0043(1)$ & - & - \\
$M_{2000}$ & $0.0018(2)$ & $0.0017(1)$ & $0.052(1)$ & - \\
$M_{5000}$ & $0.0000(1)$ & $0.00006(5)$ & $0.023(1)$ & - \\
$M_{60000}$ & - & - & - & 0 \\
\hline$d_{20}$ & $d_{100}$ & \multicolumn{5}{c}{$d_{200}$} & $d_{2000}$ & $d_{5000}$ \\
$0.0115(1)$ & $0.0046(1)$ & $0.0033(1)$ & $0.0007(1)$ & $0.00036(1)$ \\
\hline \multicolumn{5}{r}{}
\end{tabular}




\section{Discussion}

In several previous studies, FM in LCO nanoparticles has been attributed to FM ordering of the surface, [14, 15] surface-induced lattice strain, [12] and unit-cell expansion. [19, 20] Yan et al. 14] found that the magnetic susceptibility of their bulk particles increased as the surfaceto-volume ratio increased; as all of the samples were from the same single crystal sample, this indicates that surface effects increase FM in LCO. Harada et al. [15] and Fita et al. 12] found that a decrease in particle size correlates with an increase the net moment. These studies are consistent with a surface-induced tensile stress resulting from lattice expansion of the surface regions, and with tensile stress in thin films induced by substrates [16, 21 23].

To interpret the results of the fits to the magnetization and lattice parameters, we employ a core-interface model with two regions distinguished by the character of the magnetic interactions and the proximity to surfaces or interfaces. The core region of the particles, far from any surface or interface, is particularly significant in the larger particles. In this region, the dominant interaction is AFM. However, the peak seen near $T=30 \mathrm{~K}$ in the $H / M$ data for samples $\mathrm{A}$ and $\mathrm{B}$ is broad, not sharp as would be expected in the case of long-range AFM order. We thus conclude that the core region does not order antiferromagnetically.

The AFM correlations vary with $\gamma$ (or, equivalently, $\delta y$ ), which crosses a critical value at $T_{o} \approx 40 \mathrm{~K}$. The high $T$ AFM correlations in the core region disappear below $T_{o}$, and the Curie-Weiss parameters $E_{b}$ and $t_{b}$ are smaller in this temperature range.

While it might be reasonable to interpret this as a paramagnetic behavior with weaker moments and interactions, it was found [4] in the bulk particles that the extrapolation of the low $T$ Curie-Weiss expression to $T=0$ nearly coincides with the extrapolation to $T=0$ of the Curie-Weiss fit in the range $170<T<300 \mathrm{~K}$. This could indicate that the moments and antiferromagnetic interactions remain the same and the correlations are shortranged in both temperature regions. This, in turn, would imply that the system is highly frustrated for $T<T_{o}$. The core region has a large volume in samples A and B and is responsible for most of the antiferromagnetic contribution to $H / M$ for large $H$. However, the $T=0$ value of $H / M$ is significantly smaller in powder $\mathrm{B}$. This would be consistent with a smaller volume of the core region in $\mathrm{B}$ because moments are all near interfaces or surfaces. In the interface region, $\gamma$ never crosses the critical value as a result of tensile strain, and thus remains large enough to sustain long-range ferromagnetic order. [16] Interfaces with other phases, as well as the mismatch in lattice parameters of the core and interface regions, can be sources of tensile strain that can induce long-range ferromagnetic order.
In the bulk particles of sample A, core region volumes are large and peaks in $H / M$ vs $T$ for large $H$ near $T_{o}$ occur as the high $T$ antiferromagnetic paramagnetism gives way to low $T$ paramagnetism with weaker antiferromagnetic correlations. It was shown [4] that the standard procedure of growing bulk particles in ambient atmosphere results in unreacted $\mathrm{Co}_{3} \mathrm{O}_{4}$ remaining in the sample. Bulk sample $\mathrm{A}$ has about $4.5 \% \mathrm{Co}_{3} \mathrm{O}_{4}$ by weight, which could allow for $\mathrm{LCO}-\mathrm{Co}_{3} \mathrm{O}_{4}$ crystallite interfaces. In addition to the impurity phase interfaces, the weak ferromagnetic power law behavior is consistent with the relatively small volume of the interface region near particle surfaces. This power law behavior for all $H$ is followed quite well for $T_{o}<T<T_{C}$, but the ferromagnetic contribution to the magnetization at small $H$ falls significantly below the power law for $T<T_{o}$ (see Fig. 3(A)). Notably, muon depolarization [24] peaks in the temperature region $T_{o}<T<T_{C}$, are consistent with a decrease in correlations below $T_{0}$.

The exponent $\beta=0.63$ describes the power law behavior near $T_{C}$ well, but is inconsistent with expected bulk ferromagnetic long-range ordering, for which $\beta<<\frac{1}{2}$. However, Binder and Hohenberg (BH) 25, 26] have predicted an exponent, $\beta=0.65$, for surface critical behavior which is consistent with our fits and, in turn, with the ferromagnetism being associated primarily with LCO surfaces and interfaces. Notably, BH surface magnetism is assisted by the bulk ordering and would normally be difficult to observe against the ordering moment of the bulk. For LCO, it is primarily the surface that orders ferromagnetically, making it possible to observe the weak 2D ferromagnetic ordering. Seo et al. [27] suggest that the ferromagnetic moment near surfaces is a result of spin canting. If so, moments near the surface must also order antiferromagnetically and the $\mathrm{BH}$ surface ordering mechanism would be associated with 2D antiferromagnetic ordering at the surface and $3 \mathrm{D}$ antiferromagnetism further away from the surface. The weakening of the antiferromagnetism in the core region and the concomitant weakening of the ferromagnetism below $T_{o}$ indicates they may be related, i.e. the core region plays a role in supporting the surface magnetism. The weak ferromagnetic ordering in the bulk particles has yet to be observed using neutron scattering, so it is not surprising that weak antiferromagnetic ordering has also not yet been observed. LCO single crystals show behavior similar to the bulk particles, despite a small proportion of surface area [14]. This may be due to significant twinning and defects that serve a similar role as free surfaces, resulting in similar submicron structures.

The large nanoparticles of sample B show remarkably similar, but not identical, magnetic behavior to the bulk. This suggests that the relative proportions of the core and interface regions, are similar. Although the amount of $\mathrm{Co}_{3} \mathrm{O}_{4}$ is less in the large nanoparticles, the smaller size of the particles results in more particle surface area, 
which can have a similar effect.

Remarkable features of the small nanoparticles in sample $\mathrm{C}$ include the lack of a clear crossover of the antiferromagnetic behavior and a relatively weak structural signature of the transition near $T_{o}$. This suggests that core region moments occupy a relatively minor part of the sample; nearly all of the moments are close to the surface in the interface region. With the large surface area, one might expect the overall magnetic moment to be much larger than that of samples A and B. However, without strain from a mismatch of core and interface region, ferromagnetic long-range order generated by the particle surfaces is greatly reduced in the interface region.

Powder D differs primarily from $\mathrm{C}$ in the amount of $\mathrm{Co}_{3} \mathrm{O}_{4}$, which is nearly three times larger in D. Apparently, the magnetic moment can be made much larger with the introduction of strain from $\mathrm{Co}_{3} \mathrm{O}_{4}$. The particles in $\mathrm{C}$ and $\mathrm{D}$ are similar in size, but the introduction of $\mathrm{Co}_{3} \mathrm{O}_{4}$ results in even less core region volume than sample C. The lack of any apparent transition in the lattice parameters near $T_{o}$, the $\mathrm{CW}$ fits using the same temperature at large and small $T$, and the close tracking of the magnetization to the power law to low $T$ are consistent with insignificant core region volume. It has been shown that, in bulk particles, [4] the $\mathrm{LCO} / \mathrm{Co}_{3} \mathrm{O}_{4}$ interfaces cause significant ferromagnetism, presumably because they introduce tensile strain into the LCO lattice. The particles in D are nearly all in the interface region and, with the introduction of tensile strain from $\mathrm{Co}_{3} \mathrm{O}_{4}$, the ferromagnetic moment is an order of magnitude stronger than in any of the other powders.

We have shown that the various magnetic behaviors of LCO powders are consistent with the presence of two kinds of magnetic regions, the interior core region of the larger particles and the interface region near surfaces or interfaces with other phases. The literature has examples of nanoparticle LCO, some showing phase transitions and others not. 19, 20] In our model, these differences would reflect the density of interfaces with phases such as $\mathrm{Co}_{3} \mathrm{O}_{4}$. This may explain, for example, the surprising results of Wei et al. 19] which show a decrease in magnetization and $T_{C}$ with decreasing nanoparticle size. These are in contrast to the results by Fita et al. and Yan et al., which show the opposite effect. [12, 14]

This model will help in the interpretation of the magnetic behavior of large LCO crystals, which typically show large amounts of twinning, and LCO films grown on substrates. It will be useful in comparing band structure simulations with magnetic and structural data. Most importantly, it will aid the search for materials with switchable ferromagnetism that are suitable for making spintronic devices that operate above room temperature.

We thank F. Bridges, A. Elvin, B. Harmon, J. Howe, A. P. Ramirez, and N. Sundaram for helpful discussions and/or assistance with measurements.

The work at the High Flux Isotope Reactor at ORNL was supported by the DOE BES Office of Scientific User Facilities. Work at Lawrence Berkeley National Laboratory was supported by the Director, Office of Science (OS), Office of Basic Energy Sciences (OBES), of the U.S. Department of Energy (DOE) under Contract No. DEAC02-05CH11231. Some X-ray data in this work were recorded on an instrument supported by the NSF Major Research Instrumentation (MRI) Program under Grant DMR-1126845.

[1] G. E. Sterbinsky, P. J. Ryan, J.-W. Kim, E. Karapetrova, J. X. Ma, J. Shi, and J. C. Woicik, Phys. Rev. B 85, 020403 (2012).

[2] V. Gopalan and D. B. Litvin, Nature Materials 10, 376 (2011).

[3] C. Hu, K. Park, A. Posadas, J. Jordan-Sweet, A. Demkov, and E. Yu, J. Appl. Phys. 114, 183909 (2013).

[4] A. M. Durand, T. J. Hamil, D. P. Belanger, S. Chi, F. Ye, J. A. Fernandez-Baca, Y. Abdollahian, and C. H. Booth, unpublished (2014).

[5] A. M. Durand, D. P. Belanger, C. H. Booth, F. Ye, S. Chi, J. A. Fernandez-Baca, and M. Bhat, J. Phys.: Condens. Matter 25, 382203 (2013).

[6] Y. Lee and B. N. Harmon, J. Appl. Phys. 113, 17E145 (2013).

[7] R. P, P. A. Korzhavyi, H. Fjellvag, and A. Kjekshus, Phys. Rev. B 60, 16423 (1999).

[8] D. Louca, J. L. Sarroa, J. D. Thompson, H. Roder, and G. H. Kwei, Phys. Rev. B 60, 10378 (1999).

[9] Y. F. Zhu, R. Q. Tan, T. Yi, S. S. Ji, X. Y. Ye, and L. L. Cao, J. of Mat. Sci. 35, 5415 (2000).

[10] Y. Jiang, F. Bridges, N. Sundaram, D. P. Belanger, I. E. Anderson, J. F. Mitchell, and H. Zheng, Phys. Rev. B 80, 144423 (2009).

[11] J. Rodriguez-Carvajal, FULLPROF: A program for Rietveld refinement and pattern matching analysis, Powder Diffraction, satellite meeting of the XVth IUCr congress (1990).

[12] I. Fita, V. Markovich, D. Mogilyansky, R. Puzniak, A. Wisniewski, L. Titelman, L. Vradman, M. Herkowitz, V. Varyukhin, and G. Gorodetsky, Phys. Rev. B 77, 224421 (2008).

[13] A. Mineshige, M. Kobune, S. Fujii, Z. Ogumi, M. Inaba, T. Yao, and K. Kikuchi, J. Sol. St. Chem. 142, 374 (1999).

[14] J.-Q. Yan, J.-S. Zhou, and J. B. Goodenough, Phys. Rev. B 70, 014402 (2004).

[15] A. Harada, T. Taniyama, Y. Takeuchi, T. Sato, T. Kyomen, and M. Itoh, Phys. Rev. B 75, 184426 (2007).

[16] D. Fuchs, E. Arac, C. Pinta, S. Schuppler, R. Schneider, and H. v. Lohneysen, Phys. Rev. B 77, 014434 (2008).

[17] S. Park, P. Ryan, E. Karapetrova, J. W. Kim, J. X. Ma, J. Shi, J. W. Freeland, and W. Wu, Appl. Phys. Lett. 95, 072508 (2009).

[18] D. Fuchs, L. Dieterle, E. Arac, R. Eder, P. Adelmann, V. Eyert, T. Kopp, R. Schneider, D. Gerthsen, and H. v. Lohneysen, Phys. Rev. B 79, 024424 (2009).

[19] Q. Wei, T. Zhang, X. P. Wang, and Q. F. Fang, Eur. 
Phys. J. Appl. Phys. 57, 30401 (2012).

[20] S. Zhou, L. He, S. Zhao, Y. Guo, J. Zhao, and L. Shi, J. Phys. Chem. C 113, 13522 (2009).

[21] H. Liu, L. Shi, S. Zhou, J. Zhao, Y. Guo, C. Wang, and L. He, Surface and Coatings Technology 226, 108 (2013).

[22] A. Posadas, M. Berg, H. Seo, D. J. Smith, A. P. Kirk, D. Zhernokletov, R. M. Wallace, A. de Lozanne, and A. A. Demkov, Microelectronic Engineering 88, 1444 (2011).

[23] A. Herklotz, A. D. Rata, L. Schultz, and K. Dorr, Phys.
Rev. B 79, 092409 (2009).

[24] S. R. Giblin, I. Terry, D. Prabhakaran, A. T. Boothroyd, J. Wu, and C. Leighton, Phys. Rev. B 74, 104411 (2006).

[25] K. Binder and P. C. Hohenberg, Phys. Rev. B 6, 3461 (1972).

[26] K. Binder and P. C. Hohenberg, Phys. Rev. B 9, 2195 (1974).

[27] H. Seo, A. Posadas, and A. A. Demkov, Phys. Rev. B 86, 014430 (2012). 